\title{
ARTICLE
}

Received 11 Oct 2012 | Accepted 14 Feb 2013 | Published 19 Mar 2013

DOI: 10.1038/ncomms2601

\section{Phase-locked indistinguishable photons with synthesized waveforms from a solid-state source}

\author{
Clemens Matthiesen ${ }^{1}$, Martin Geller ${ }^{1,2}$, Carsten H.H. Schulte ${ }^{1}$, Claire Le Gall, Jack Hansom ${ }^{1}$, Zhengyong Li ${ }^{3}$, \\ Maxime Hugues ${ }^{4}$, Edmund Clarke $^{4} \&$ Mete Atatüre ${ }^{1}$
}

Resonance fluorescence in the Heitler regime provides access to single photons with coherence well beyond the Fourier transform limit of the transition, and holds the promise to circumvent environment-induced dephasing common to all solid-state systems. Here we demonstrate that the coherently generated single photons from a single self-assembled InAs quantum dot display mutual coherence with the excitation laser on a timescale exceeding $3 \mathrm{~s}$. Exploiting this degree of mutual coherence, we synthesize near-arbitrary coherent photon waveforms by shaping the excitation laser field. In contrast to post-emission filtering, our technique avoids both photon loss and degradation of the single-photon nature for all synthesized waveforms. By engineering pulsed waveforms of single photons, we further demonstrate that separate photons generated coherently by the same laser field are fundamentally indistinguishable, lending themselves to the creation of distant entanglement through quantum interference.

\footnotetext{
${ }^{1}$ Cavendish Laboratory, University of Cambridge, Cambridge CB3 OHE, UK. ${ }^{2}$ Fakultät für Physik and CENIDE, Universität Duisburg-Essen, 47048 Duisburg, Germany. ${ }^{3}$ Key Laboratory of Luminescence and Optical Information of Ministry of Education, Beijing Jiaotong University, Beijing 100044, China. ${ }^{4}$ EPSRC National Centre for III-V Technologies, University of Sheffield, Sheffield S1 3JD, UK. Correspondence and requests for materials should be addressed to M.A. (email: ma424@cam.ac.uk).
} 
S ignificant progress has been reported within quantum information science for quantum-dot (QD) spins as stationary qubits ${ }^{1,2}$, including state preparation ${ }^{3,4}$, long spin-coherence times ${ }^{5,6}$, ultrafast optical manipulation capabilities $^{7,8}$ and single-shot read-out ${ }^{9}$. A successful realization of a solid-state quantum network relies on scalable entangling gates between individual spins. Non-local entanglement based on photon quantum interference ${ }^{10}$ has been identified as the most promising and robust approach ${ }^{11}$. The robustness stems from the probabilistic nature of entanglement creation, placing fewer constraints on detection efficiencies, but relies crucially and fundamentally on indistinguishable photons from separate spins. For QDs, the quality of photons as flying qubits, however, has remained systematically below par due to detrimental effects of the solid-state environment on the photon generation process, casting a major challenge on this roadmap today.

Resonance fluorescence, recently demonstrated for selfassembled InAs QDs ${ }^{12-15}$, avoids optically exciting the host material and therefore suppresses these dynamics; however, the ambient charge fluctuations, for example, can still broaden a transition via spectral wandering ${ }^{16}$. Deviation from a transformlimited photon wavepacket renders the use of QDs in a quantum optical network problematic, necessitating further progress in understanding these dynamics, as well as suppressing their effects. Elastic scattering from an optical transition ${ }^{17}$ represents an alternative approach to high-quality photon generation, which avoids significant population in dephasing-prone excited states. Unlike the spontaneously emitted photons, the elastically scattered photons in the Heitler regime are phase-locked to the excitation laser with ideally infinite mutual coherence, accentuating their potential for quantum interference ${ }^{18}$. In wellisolated experimental systems, such as trapped atoms and ions, this mutual coherence was observed via an optical heterodyning technique ${ }^{19}$, which allowed studying the broadening of elastic scattering owing to motional and vibrational dynamics ${ }^{20,21}$, when the species are driven by a single frequency laser. For semiconductor QDs elastic scattering in resonance fluorescence was observed recently ${ }^{22,23}$, ruling out strong fast dephasing of the optical transition. The effect of environment dynamics, apparent through spectral wandering for instance, can still limit the mutual coherence between the excitation laser and the photons generated in the Heitler regime.

Here, we demonstrate the suitability of coherently scattered QD photons as flying qubits in quantum optical networks. First, phase-sensitive heterodyning measurements are employed to determine the extent to which phase locking to the excitation laser occurs. The sub-hertz mutual coherence indicates that spectral diffusion is not significant in our sample on the measurement timescale and allows us to synthesize the waveform of the coherently scattered single photons. The accessible bandwidth for waveform synthesis is restricted only by the mutual coherence for low frequencies, and the QD transition linewidth for high frequencies. Finally, we demonstrate through two-photon interference (TPI) that the coherent QD photons are fundamentally indistinguishable, attesting their potential in quantum networks.

\section{Results}

Mutual coherence of QD photons and the excitation laser. In the experiments reported here we excite resonantly and collect the resonance fluorescence from the neutral exciton transition of InAs/GaAs self-assembled QDs embedded in a Schottky heterostructure at $4.2 \mathrm{~K}$ (see Methods). The resonance fluorescence is isolated from the excitation laser with a signal-to-background ratio better than 100:1 using a cross-polarization technique ${ }^{14,22}$.
The QD transition is Stark-tunable by $80 \mathrm{GHz}$ around $315,315 \mathrm{GHz}(951 \mathrm{~nm})$. Spectral wandering broadens the $2 \pi \times 250 \mathrm{MHz}$ radiative linewidth noticeably only on timescales of seconds. Figure 1a shows the experimental arrangement used to perform optical heterodyning measurements (see Methods). QD resonance fluorescence at frequency $v$ is superimposed with a strong local oscillator originating from the excitation laser but shifted in frequency by $\delta v \approx 210 \mathrm{kHz}$. This frequency difference results in a beat note in the difference signal of two balanced photodiodes (PD1,2). A spectrum analyser calculates the power spectrum via a fast Fourier transform algorithm. The linewidth and the lineshape of the power spectrum reflect directly the phase stability, that is, the mutual coherence between the two fields during the course of the measurement. In particular, periodic dynamics of the relative phase appear as sidebands and aperiodic fluctuations appear as broadening of the spectrum. Figure $1 \mathrm{~b}$ displays a representative raw spectrum with a strong peak at the beating frequency. The inset shows that a Gaussian lineshape with a full-width-at-half-maximum, $\Delta v_{\mathrm{FWHM}}$, of $299 \pm 9 \mathrm{mHz}$ fits the data of 5-s continuous acquisition. This raw spectrum is dominated by the system response resolution of $200 \mathrm{mHz}$ for the measurement settings. A longer acquisition time yields higher spectral resolution, hence narrower linewidths (for example, $172 \pm 12 \mathrm{mHz}$ for 10 -s acquisition time), but phase fluctuations induced by the mechanical instability of the setup reduce the signal quality and limit the obtainable linewidth (see Supplementary Note 1 and Supplementary Fig. S1). Nevertheless, even these raw linewidths indicate that every photon is phase-locked to the excitation laser with mutual-coherence time exceeding $3 \mathrm{~s}$, which corresponds to a mutual-coherence length of one million kilometres. These results confirm the prediction for an ideal twolevel system that each photon inherits fully the coherence
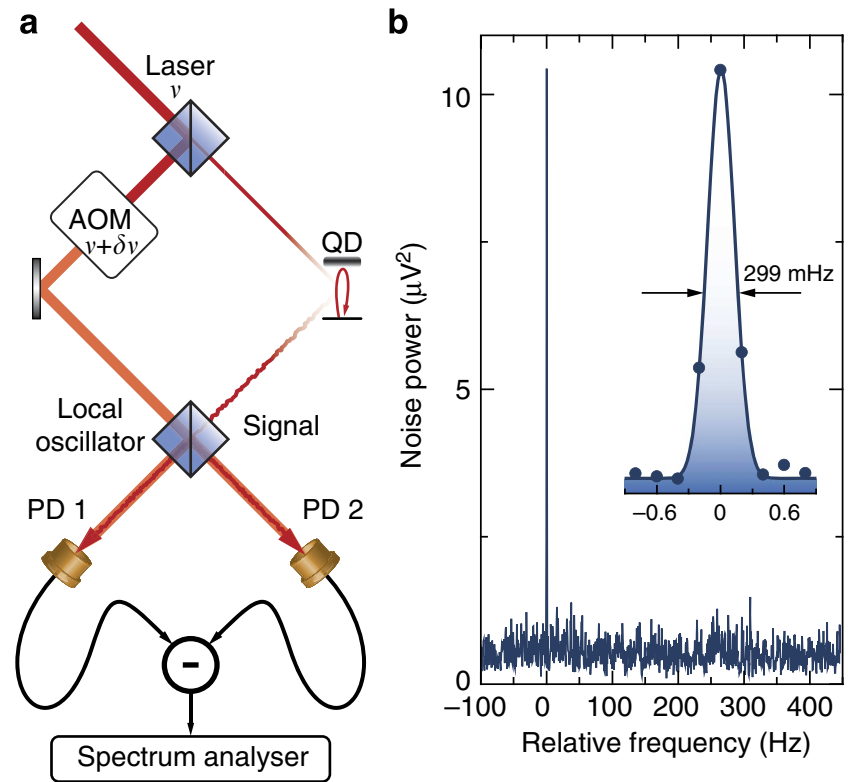

Figure 1 | Measurement of mutual coherence using optical heterodyning. (a) An illustration of the experimental arrangement used for optical heterodyning of QD resonance fluorescence. Acousto-optical modulation ( $A O M$ ) provides the shift of the local oscillator frequency by $\delta v \approx 210 \mathrm{kHz}$. The outputs of the two photodiodes (PD1,2) are subtracted electrically and sent to a spectrum analyser. (b) Typical power spectrum of the beating signal as a function of frequency relative to $\delta v$. Inset: high-resolution spectrum for $5 \mathrm{~s}$ of continuous data acquisition. Spectra with sub-hertz resolution reveal phase coherence between QD photons and excitation laser on a timescale of seconds. 
properties of the excitation laser in this alternative photon generation process. The absence of linewidth broadening beyond our detection limits further verifies that spectral diffusion occurs on timescales of seconds or slower in our experiment, which will permit feedback to stabilize the transition, for example, using electric fields ${ }^{24}$.

Phase shaping of single-photon waveforms. Methods currently available to control single-photon wavepackets range from direct spectral filtering ${ }^{25}$ to intra-photon phase encoding via photon transmission through electro-optic elements ${ }^{26-28}$. Wavepacket control during the photon generation process has only been achieved for trapped atoms inside optical cavities using multipulse sequences ${ }^{29,30}$. The coherent nature of elastic scattering provides a means for coherent synthesis of single-photon waveforms directly in the photon generation process without the need for spectral filtering or optical cavities. In order to demonstrate this ability, we use an electro-optic modulator driven by a variable amplitude $200 \mathrm{MHz}$ radio-frequency source (see Supplementary Methods) to encode the excitation laser field. Figure 2 presents two examples of coherent control of single-photon waveforms by this technique. The first example of a synthesized laser waveform and the corresponding spectrum are displayed in Fig. 2a,b, respectively. The temporal measurement in Fig. 2a is performed using a PD with $8 \mathrm{GHz}$ bandwidth, whereas the spectrum in Fig. $2 \mathrm{~b}$ is measured using a stabilized FabryPerot interferometer (see Methods). Figure 2c,d displays another example for encoding the laser field with a more complicated pattern, where the component at the carrier frequency (zero detuning) is strongly suppressed. The single-photon QD emission spectra for the two examples are presented in Fig. 2e,f. The 200$\mathrm{MHz}$ spectral spacing and the relative strength of the frequency components are dictated by the laser spectrum for each example, in addition to being weighted by the QD transition linewidth. The radiative linewidth for a measured excited state lifetime of $0.65 \mathrm{~ns}$ is indicated as grey shaded areas in panels e and $\mathrm{f}$ for comparison. In both cases, the strong antibunching observed in the intensity-correlation measurements is fully sustained (see Supplementary Note 2 and Supplementary Fig. S2). We note that although these examples are based on amplitude modulation of the laser field, encoding phase to each spectral component is possible in this scheme. These measurements demonstrate that, as a consequence of their mutual coherence with the laser field, the single-photon waveforms can be synthesized deterministically without post-generation filtering, which can lead to photon loss and degradation of the singlephoton quality.

Indistinguishability of pulsed coherent single photons. A key requirement of a photonic link in a quantum network is time-synchronized indistinguishable single-photon streams. However, the uncorrelated environment fluctuations experienced by separate QDs under non-resonant excitation lead to variations in temporal and spectral overlap of photon wavepackets ${ }^{31,32}$. Imparting mutual coherence on independent photons via a common excitation laser can therefore be a crucial advantage in achieving indistinguishable wavepackets. Therefore, we perform Hong-Ou-Mandel (HOM)-style TPI experiments ${ }^{33}$ of two QD photons scattered from the same QD at different times to quantify the indistinguishability ${ }^{34}$ of synthesized photon waveforms with complex spectra. A schematic of the experimental setup is shown in Fig. 3a. Figure 3b displays the laser pulse train (top panel) used to generate coherent QD photons in the Heitler regime (bottom). The laser pulses are derived from a continuous wave laser by modulating the electro-optic modulator transmission with independent control on the duration and the a

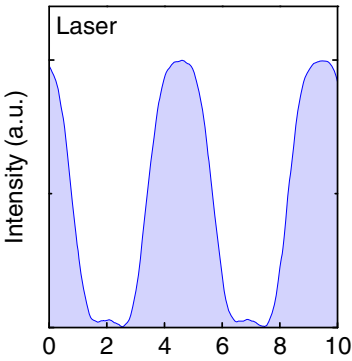

c

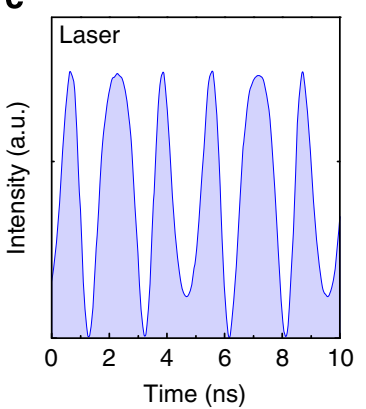

b

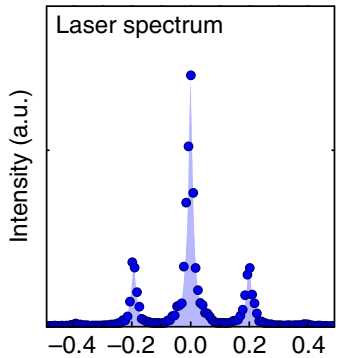

d

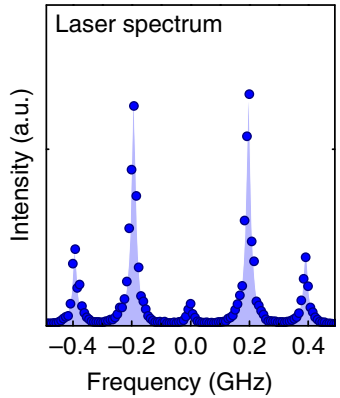

e

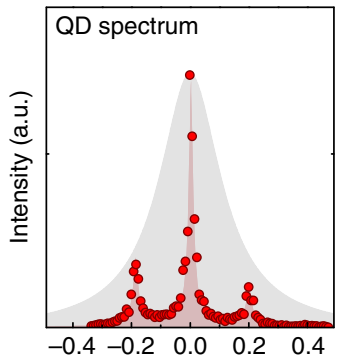

f

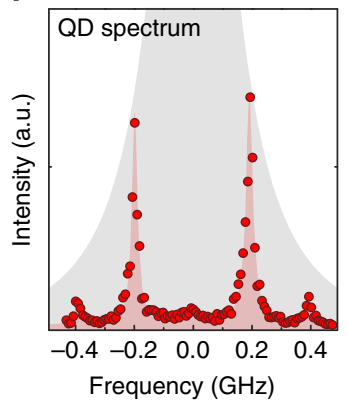

Figure 2 | Imprinting arbitrary waveforms on single photons. (a) The intensity of the excitation laser is modulated in time using an electro-optic modulator driven with a 200-MHz sine wave. (b) The spectrum of the modulated laser, measured through a Fabry-Perot cavity (resolution $\sim 20 \mathrm{MHz}$ ), shows sidebands at the modulation frequency. (c,d) A different waveform modulation with the corresponding spectrum for the laser. This waveform is designed to suppress the spectral component at the original carrier frequency. (e,f) Measured spectra of the QD photons generated from the two synthesized laser waveforms given in $\mathbf{a}, \mathbf{c}$, respectively. The elastically scattered photons replicate the laser spectrum within the linewidth of the transition, which is indicated by the shaded grey area. 
a

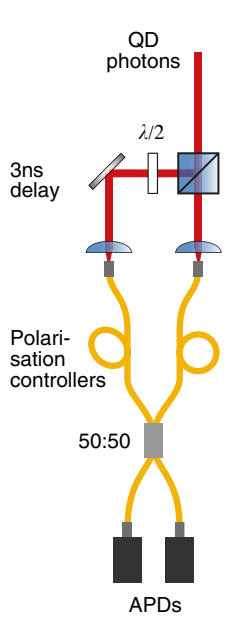

b

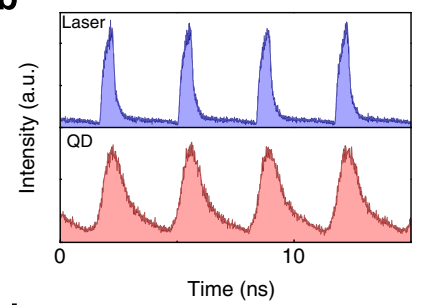

d

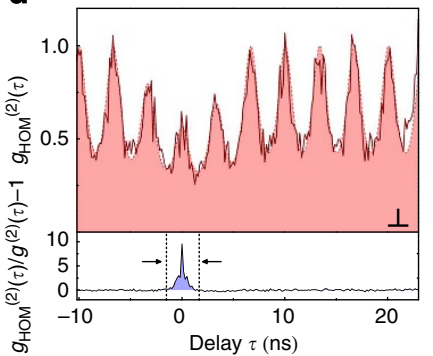

c
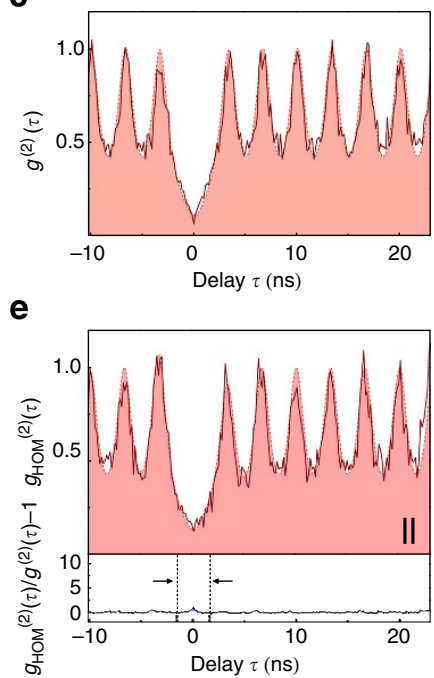

Figure 3 | Indistinguishability of waveform-synthesized photons. (a) Illustration of the experimental setup for two-photon interference from a single QD. (b) Time-synchronized single QD photons (bottom panel) are generated by exciting the QD transition with laser pulses of 500-ps width and 300-MHz repetition rate (top panel). (c) The intensity autocorrelation function for QD photons is recorded in a Hanbury-Brown and Twiss setup. A two-photon probability per pulse below $5 \%$ demonstrates the quantum nature of the emitter. (d,e) Two-photon interference (TPI) measurements for QD photons of orthogonal (d) and parallel (e) polarization. The bottom panels display the normalized differences to the intensity autocorrelation in c, respectively. The lack of TPI for orthogonally polarized photons in $\mathbf{d}$ gives rise to coincidences at zero time delay, yielding a peak in the bottom panel. For photons of parallel polarization, the absence of coincidences around zero time delay reflects successful TPI in $\mathbf{e}$. The ratio of the areas in the bottom panels yields a raw visibility of 0.926 . Experimental data in c-e are shown as continuous dark red curves, while a simulation for each case based on system parameters is shaded in light red. The data are recorded in 162-ps time bins with 600-ps timing resolution.

repetition rate (see Supplementary Fig. S3). Here, the laser pulses are 500-ps long with a repetition rate of $300 \mathrm{MHz}$. For these settings the temporal response of the QD transition is visible as an exponential tail in the coherent conversion of laser pulses into single photons. Figure $3 \mathrm{c}$ shows the intensity-correlation measurement, that is, $g^{(2)}(\tau)$, for these photons. The missing peak at zero time delay in coincidence detection evidences strong antibunching. For pure photonic states, a HOM-style TPI measurement in the vicinity of zero time delay is expected to follow

$$
g_{\mathrm{HOM}}^{(2)}(\tau)=\left(T_{1}^{2}+R_{1}^{2}\right) g^{(2)}(\tau)+2 R_{1} T_{1}\left(1-\eta+\eta g^{(2)}(\tau)\right),
$$

where $T_{1}$ and $R_{1}$ are the transmission and reflection coefficients of the first beam-splitter used to form the two input arms of the second beam-splitter (cf. Fig. 3a). The variable $\eta$ quantifies the photon indistinguishability including any imperfections of the measurement apparatus. For fully indistinguishable $(\eta=1)$ photons $g_{\mathrm{HOM}}^{(2)}(\tau)$ reduces to $g^{(2)}(\tau)$, while for fully distinguishable $(\eta=0)$ photons equation (1) takes the form $g_{\text {HOM }}^{(2)}(\tau)=\left(T_{1}^{2}+R_{1}^{2}\right) g^{(2)}(\tau)+2 R_{1} T_{1}$. We use the polarization of the input photons as means to vary $\eta$, such that parallel (orthogonal) polarization leads to a maximum (minimum) value for $\eta$. These measurements, performed in conditions identical to those of Fig. 3c, are shown in Fig. 3d,e for orthogonal and parallel input polarization states, respectively. To quantify the TPI fidelity, we compare the data directly with the ideal case of $g_{\mathrm{HOM}}^{(2)}(\tau)=g^{(2)}(\tau)$ : the bottom panels of Fig. 3d,e show $\left(g_{\mathrm{HOM}}^{(2)}(\tau) / g^{(2)}(\tau)-1\right)$ for their respective HOM measurement. Integrating this normalized difference curve over one repetition period $(\sim 3.3 \mathrm{~ns})$ centred around zero time delay, we can extract a raw contrast of the TPI experiment of $C_{\mathrm{HOM}}=1-\left(A_{\mathrm{pa}} / A_{\text {or }}\right)=0.926 \pm 0.016$. Taking into account deviations from ideal experimental conditions, for example, imperfect polarization control and unbalanced interferometer arms, in the conventional way ${ }^{34}$ we extract a corrected contrast of unity within the experimental uncertainty $\left(C_{\text {cor }}=1.03 \pm 0.05\right)$. However, the correction for the unbalanced interferometer is complicated by the coherence of the photons sustained beyond the interferometer delay (see Supplementary Notes 3 and 4 and Supplementary Fig. S4). Therefore, limiting the corrections to only those arising from imperfect polarization control we report a lower bound on photon wavepacket indistinguishability of $0.96 \pm 0.04$. This value surpasses all reports on spontaneously emitted QD photons to-date and confirms the expected advantage of the coherently scattered photons replicating the laser coherence. A TPI measurement for higher excitation power is presented in Supplementary Fig. S5. We stress that the lack of correlation between the environment dynamics of multiple QDs will inevitably lead to the degradation of photon indistinguishability for the incoherently generated photons as is evident in all attempts to date $\mathrm{e}^{31,32}$, while the coherent generation process reported here ensures the immunity of our timesynchronized coherent photon wavepackets to such effects.

\section{Discussion}

We have demonstrated the generation of fundamentally indistinguishable, coherent single photons from a solid-state quantum emitter with a high degree of control and flexibility in waveform synthesis. These phase-locked photons are ideally suited for quantum interference applications, which form the basis of quantum communication ${ }^{18}$, linear optics quantum computation $^{35}$ and distant entanglement schemes ${ }^{10,11}$. The price to pay for this unprecedented photon quality is excitation efficiency. To obtain a coherent fraction of about 0.9 in pulsed excitation, photons are probabilistically generated with 5-10\% efficiency $\left(1.5-3 \times 10^{7}\right.$ photons per second for the HOM 
measurements presented here). Although generation rates are lower than those achievable in deterministic excitation schemes, the trade-off between photon rates and photon quality is well justified for two main reasons: first, quantum interference applications can work well with probabilistic gates ${ }^{11,36}$, while the low photon quality reported so far ${ }^{31,32}$ rules out the use of QD photons in quantum information applications. Further, non-unity photon collection and detector efficiencies evidently render any measurement-based schemes probabilistic. Second, an entanglement rate scaling with the two-photon detection efficiency ${ }^{11}$ is replaced advantageously by one-photon detection ${ }^{10,18}$, utilizing the first-order coherence of the coherently scattered photons. The technique presented in the work is not limited to QDs and can be extended to other quantum systems in the optical domain as well as to superconducting circuits in the microwave domain. Spinselective transitions of QDs will be utilized next to transfer the quantum mechanical state of a spin to frequency and polarization degrees of freedom of a photon. The ability to tailor singlephoton waveforms is also advantageous in efficiently storing information in quantum memories with restricted spectral bandwidth. Building on these results, we aim to realize a distributed network of multiple QD spins interfaced with waveform-synthesized and time-synchronized single photonsall phase-locked to a master laser.

\section{Methods}

QD sample structure. We use a sample grown by molecular beam epitaxy containing a single layer of self-assembled InAs/GaAs QDs in a GaAs matrix and embedded in a Schottky diode for charge state control. The Schottky diode is formed by gating an $\mathrm{n}+$-doped layer $35 \mathrm{~nm}$ below the QDs with a 5-6-nm thick partially transparent titanium layer evaporated on top of the sample surface. This device structure allows for deterministic charging of the QDs and shifting of the QD exciton energy levels via the DC Stark effect. Twenty pairs of GaAs/AlGaAs layers forming a distributed Bragg reflector extend to $205 \mathrm{~nm}$ below the QD layer for increased collection efficiency in the spectral region between 960 and $980 \mathrm{~nm}$. Spatial resolution and collection efficiency are enhanced by a zirconia solid immersion lens in Weierstrass geometry positioned on the top surface of the device. The measurements reported here are repeated on two separate QDs for further confirmation. For both QDs the neutral exciton transition (one transition of the exchange doublet) was optically addressed. The wavelength of this transition was at $951 \mathrm{~nm}$ for both QDs.

Resonance fluorescence measurement technique. Resonant optical excitation and collection of the fluorescence from single QDs is achieved using a confocal microscope with 10:90 beam-splitter head in a 4.2-K liquid helium bath cryostat. We excite and collect both QD fluorescence and laser scattering along the same path via a $0.4 \mathrm{NA}$ objective lens inside the cryostat. The QD transition dipole has a preferential in-plane orientation, independent of excitation orientation and polarization, therefore we excite in a linear polarization at $\sim 45^{\circ}$ to the transition polarization and collect in the orthogonal polarization. This ideally removes all laser at the cost of filtering half of the scattered QD photons. In practice, $10^{7}$ suppression of the laser reflection is achieved combining this cross-polarized detection with confocal rejection due to coupling to a single-mode fibre. The contribution of the phonon sideband represents $\sim 12 \%$ of the total QD emission in our sample. For TPI measurements, the QD fluorescence is spectrally filtered via a $1,600-\mathrm{g} \mathrm{mm}^{-1}$ grating acting as a bandpass filter for the zero-phonon line. The experimental setup including the dark field detection scheme and the spectral filtering, together with an unfiltered emission spectrum is illustrated in Supplementary Fig. S6. The laser background-to-signal ratio is well below $1 \%$ for all measurements presented. Under pulsed excitation, the excitation power was chosen so that the elastically scattered photons exceed $90 \%$ of the total fluorescence (see Supplementary Note 3 and Supplementary Fig. S4). Working in this regime yields approximately one scattering event per 20 excitation pulses.

Optical heterodyning of QD resonance fluorescence. A continuous wave laser at optical frequency $v$ is split into two beams of different intensities where the weak laser beam excites the QD. The strong laser beam is shifted in frequency to $v+\delta v$ using acousto-optical modulation. In these experiments $\delta v=210 \mathrm{kHz}$, achieved by frequency shifting the laser twice using two acousto-optical modulation devices operating at 80.000 and $79.790 \mathrm{MHz}$. This avoids any residual electronic pickup noise in the experiment in the spectral region of interest. The fields of the coherently scattered single photons $\vec{E}_{\mathrm{S}}$ (signal) and the frequency-shifted laser $\vec{E}_{\mathrm{LO}}$ (local oscillator) are combined via a beam-splitter and detected by two balanced PDs (PD1,2 in Fig. 1). The total optical intensity $I_{i}$ on each PD contains a phaseinvariant component comprising the sum of the two input intensities and a phasedependent component oscillating at the difference frequency $\delta v . I_{i}$ has the mathematical form

$$
I_{i}=\left|\vec{E}_{\mathrm{S}}+\vec{E}_{\mathrm{LO}}\right|^{2}=\left|\vec{E}_{\mathrm{S}}\right|^{2}+\left|\vec{E}_{\mathrm{LO}}\right|^{2}+2 \vec{E}_{\mathrm{S}} \vec{E}_{\mathrm{LO}} \cos \left(\delta v \cdot t+\varphi_{i}(t)\right),
$$

where $\varphi_{i}(t)$ is the relative phase between the two optical fields on $\mathrm{PD}_{i}$ at time $t$. Working with the difference of the PD signals suppresses the constant components in equation (2), while enhancing the beating signal amplitude. The PDs used for this measurement have $500 \mathrm{kHz}$ detection bandwidth. The resultant power spectrum is governed by the dynamics of the relative phase, $\varphi_{i}(t)$, in the course of the measurement. For a Gaussian lineshape the width of the power spectrum, $\Delta v_{\text {FWHM }}$ can be converted into a mutual-coherence time, $\tau_{c}$, via the relation $\tau_{\mathrm{c}}=\sqrt{2 \ln 2 / \pi} / \Delta v_{\mathrm{FWHM}} \approx 0.66 / \Delta v_{\mathrm{FWHM}}$. The mutual-coherence length is obtained using $c \times \tau_{\mathrm{c}} \approx 0.66 \times c / \Delta v_{\mathrm{FWHM}}$.

Details of the HOM interferometer. To observe TPI, we split the stream of QD photons into two arms on a nominally 50:50 beam-splitter (measured $\left.T_{1} / R_{1} \sim 1.3\right)$. The delay between the two arms $\left(\Delta t_{\text {delay }}=3.33 \mathrm{~ns}\right)$ is adjusted so that two consecutive pulses overlap temporally at the second beam-splitter $\left(T_{2}=0.41 \pm 0.01, R_{2}=0.59 \pm 0.01\right)$. The latter is fibre-based (single mode), which ensures spatial mode matching, but requires additional care for conserving the polarization of the input photons. We use custom-made polarization controllers and half-wave plates to match the input polarizations. The half-wave plate shown in Fig. 3 allows us to perform TPI measurements for photons with orthogonal and parallel polarizations with the same setup. The degree of polarization control in the interferometer is measured separately before each measurement for the two halfwave plate settings corresponding to interference of parallel and orthogonal polarizations, respectively, by sending through a narrowband single-mode laser For the measurements presented in Fig. 3, we match polarization in the interferometer to $p_{\mathrm{pa}}=0.97 \pm 0.02$ in the parallel case and measure a contrast of $p_{\text {or }}=0.05 \pm 0.02$ in the orthogonal case.

Instrument properties. The laser-stabilized Fabry-Perot cavity has a resolution of $20 \mathrm{MHz}$ and the two-photon detection system used for intensity-correlation as well as HOM interference measurements has a temporal resolution of $600 \mathrm{ps}$. The lasers are stabilized to $<2 \mathrm{MHz}$ linewidth over few hours. Uncertainties stated for the interference contrast represent 1 s.d. and are based on propagation of uncorrelated random errors.

\section{References}

1. Loss, D. \& DiVincenco, D. P. Quantum computation with quantum dots. Phys Rev. A 57, 120-128 (1998).

2. Imamoglu, A. et al. Quantum information processing using quantum dot spins and cavity QED. Phys. Rev. Lett 83, 4204-4207 (1999).

3. Atatüre, M. et al. Quantum-dot spin-state preparation with near-unity fidelity. Science 312, 551-553 (2006).

4. Gerardot, B. D. et al. Optical pumping of a single hole spin in a quantum dot. Nature 451, 441-444 (2008).

5. Xu, X. et al. Optically controlled locking of the nuclear field via coherent dark-state spectroscopy. Nature 459, 1105-1109 (2009).

6. Press, D. et al. Ultrafast optical spin echo in a single quantum dot. Nat. Photon. 4, 367-370 (2009).

7. Berezovsky, J., Mikkelsen, M. H., Stoltz, N. G., Coldren, L. A. \& Awschalom, D. D. Picosecond coherent optical manipulation of a single electron spin in a quantum dot. Science 320, 349-352 (2008).

8. Press, D., Ladd, T. D., Zhang, B. \& Yamamoto, Y. Complete quantum control of a single quantum dot spin using ultrafast optical pulses. Nature 456, 218-221 (2008).

9. Vamivakas, A. N. et al. Observation of spin-dependent quantum jumps via quantum dot resonance fluorescence. Nature 467, 297-300 (2010).

10. Cabrillo, C., Cirac, J. I., Garcia-Fernandez, P. \& Zoller, P. Creation of entangled states of distant atoms by interference. Phys. Rev. A 59, 1025-1033 (1999).

11. Duan, L.-M., Madsen, M. J., Moehring, D. L., Maunz, P., Kohn, Jr R. N. \& Monroe, C. Probabilistic quantum gates between remote atoms through interference of optical frequency qubits. Phys. Rev. A 73, 062324 (2006).

12. Muller, A. et al. Resonance fluorescence from a coherently driven semiconductor quantum dot in a cavity. Phys. Rev. Lett. 99, 187402 (2007)

13. Melet, R. et al. Resonant excitonic emission of a single quantum dot in the Rabi regime. Phys. Rev. B 78, 073301 (2008).

14. Vamivakas, A. N. et al. Spin-resolved quantum-dot resonance fluorescence. Nat. Phys 5, 198-202 (2009)

15. Flagg, E. B. et al. Resonantly driven coherent oscillations in a solid-state quantum emitter. Nat. Phys. 5, 203-207 (2009).

16. Houel, J. et al. Probing single-charge fluctuations at a GaAs/AlAs interface using laser spectroscopy on a nearby InGaAs quantum dot. Phys. Rev. Lett. 108, 107401 (2012). 
17. Heitler, W. The Quantum Theory of Radiation (Dover Publications, 2010).

18. Childress, L., Taylor, J. M., Sørensen, A. S. \& Lukin, M. D. Fault-tolerant quantum communication based on solid-state photon emitters. Phys. Rev. Lett. 96, 070504 (2006).

19. Höffges, J. T., Baldauf, H. W., Eichler, T., Helmfrid, S. R. \& Walther, H. Heterodyne measurement of the resonance fluorescence of a single ion. Opt. Comm. 133, 170-174 (1997).

20. Jessen, P. S. et al. Observation of quantized motion of Rb atoms in an optical field. Phys. Rev. Lett 69, 49-52 (1992).

21. Raab, Ch., Eschner, J., Bolle, J., Oberst, H., Schmidt-Kaler, F. \& Blatt, R. Motional sidebands and direct measurement of the cooling rate in the resonance fluorescence of a single trapped ion. Phys. Rev. Lett. 85, 538-541 (2000).

22. Matthiesen, C., Vamivakas, A. N. \& Atatüre, M. Subnatural linewidth single photons from a quantum dot. Phys. Rev. Lett. 108, 093602 (2012).

23. Nguyen, H. S. et al. Ultra-coherent single photon source. Appl. Phys. Lett. 99, 261904 (2011).

24. Acosta, V. M. et al. Dynamic stabilization of the optical resonances of single nitrogen-vacancy centers in diamond. Phys. Rev. Lett. 108, 206401 (2012).

25. Polyakov, S. V. et al. Coalescence of single photons emitted by disparate single-photon sources: the example of InAs quantum dots and parametric down-conversion sources. Phys. Rev. Lett. 107, 157402 (2011).

26. Kolchin, P., Belthangady, C., Du, S., Yin, G. Y. \& Harris, S. E. Electro-optic modulation of single photons. Phys. Rev. Lett. 101, 103601 (2008).

27. Specht, H. P. et al. Phase shaping of single-photon wave packets. Nat. Photon. 3, 469-472 (2009)

28. Rakher, M. T. \& Srinivasan, K. Subnanosecond electro-optic modulation of triggered single photons from a quantum dot. Appl. Phys. Lett. 98, 211103 (2011).

29. McKeever, J. et al. Deterministic generation of single photons from one atom trapped in a cavity. Science 303, 1992-1994 (2004).

30. Keller, M., Lange, B., Hayasaka, K., Lange, W. \& Walther, H. Continuous generation of single photons with controlled waveform in an ion-trap cavity system. Nature 431, 1075-1078 (2004).

31. Flagg, E. B. et al. Interference of single photons from two separate semiconductor quantum dots. Phys. Rev. Lett. 104, 137401 (2010).

32. Patel, R. B. et al. Two-photon interference of the emission from electrically tunable remote quantum dots. Nat. Photon. 4, 632-635 (2010).
33. Hong, C. K., Ou, Z. Y. \& Mandel, L. Measurement of subpicosecond time intervals between two photons by interference. Phys. Rev. Lett. 59, 2044-2046 (1987).

34. Santori, C., Fattal, D., Vuckovic, J., Solomon, G. S. \& Yamamoto, Y. Indistinguishable photons from a single-photon device. Nature 419, 594-597 (2002).

35. O’Brien, J. L., Furusawa, A. \& Vuckovic, J. Photonic quantum technologies. Nat. Photon. 3, 687-695 (2009).

36. Duan, L.-M. \& Raussendorf, R. Efficient quantum computation with probabilistic quantum gates. Phys. Rev. Lett. 95, 080503 (2005).

\section{Acknowledgements}

This work was supported by grants and funds from the University of Cambridge, EPSRC Science and Innovation Awards, the QIPIRC and EPSRC grant number EP/G000883/1. M.G. acknowledges the German Research Foundation (DFG) for financial support (grant number GE 2141/2-1) and Z.L. acknowledges the support of NSFC (grant number 60907027). We thank A.N. Vamivakas, J.M. Taylor, C.-Y. Lu, R.T. Phillips and A.J. Ferguson for fruitful discussions, as well as technical assistance.

\section{Author contributions}

C.M., M.G., C.H.H.S., C.L.G., J.H., Z.L. and M.A. contributed to conception, instrumentation, measurements and analysis. M.H. and E.C. grew the sample. All authors discussed the results and contributed to writing the manuscript.

\section{Additional information}

Supplementary Information accompanies this paper at http://www.nature.com/ naturecommunications

Competing financial interests: The authors declare no competing financial interests.

Reprints and permission information is available online at http://npg.nature.com/ reprintsandpermissions/

How to cite this article: Matthiesen, C. et al. Phase-locked indistinguishable photons with synthesized waveforms from a solid-state source. Nat. Commun. 4:1600 doi: $10.1038 /$ ncomms2601 (2013). 